\title{
Surface electromyography hand motion classification using time domain features and artificial neural network for real time application
}

\begin{abstract}
This paper presents the efficiency of time domain features and Artificial Neural Network (ANN) classifier for real time Surface Electromyography (SEMG) hand motion classification application in terms of real time delay and classification accuracy. For hand motion to be differentiated, SEMG data goes through pre-processing, feature extraction and classification steps. Data were collected from 10 healthy subjects. Two muscles were assessed which are flexor carpi ulnaris (FCU) and extensor carpi radialis (ECR) during 3 hand motions of wrist flexion (WF), wrist extension (WE) and co-contraction (CC) for 3 repetitions. The SEMG signals was first segmented into $132.5 \mathrm{~ms}$ window, full wave rectified and with a parallel process of comparing raw signal and a filtered signal using a $6 \mathrm{~Hz}$ low pass Butterworth filter. Five time domain features of mean absolute value (MAV), variance (VAR), root mean square (RMS), integrated absolute value (IEMG) and waveform length (WL) are used for feature extraction. Feed Forward Neural Network with 10 hidden neurons and 3 output neurons is used as classifier. First 2 repetitions of motion were used for training while the last repetition was used for classification testing. Features were added from 1 to 5 and tested for classification accuracy and computational times. Simulation computational times were recorded at pre-processing, feature extraction and classification steps. Meanwhile, hardware computational steps were recorded at pre-processing and feature extraction steps. It can be concluded that time domain features are efficient for real time application with $6 \mathrm{~ms}$ simulation delay. However, only MAV, IEMG, and WL is suitable for hardware with $68.6 \mathrm{~ms}$ delay. Meanwhile, the ANN classifier is unsuitable for real time application with $268 \mathrm{~ms}$ delay despite having achieving good accuracy of $92 \%$.
\end{abstract}

Keyword: Artificial neural network; Computational times; Time domain features 